\title{
Associação do methimazole e do ondansetron à quimioterapia com cisplatina em cães submetidos a quatro diferentes protocolos de fluidoterapia
}

\author{
[Association of methimazole and ondansetron to cisplatin chemotherapy in dogs submitted to four different fluid \\ therapy protocols] \\ C.S.F. Repetti ${ }^{1}$, C.R. Daleck ${ }^{2}$ \\ ${ }^{1}$ Faculdade de Medicina Veterinária - UNIMAR \\ Rua José Ferreira da Costa, 241 \\ 17525-902 - Marília, SP \\ ${ }^{2}$ Faculdade de Ciências Agrárias e Veterinárias - UNESP - Jaboticabal, SP
}

\begin{abstract}
RESUMO
Utilizaram-se 12 cães, machos, distribuídos em quatro grupos (G) experimentais, selecionados de acordo com o tempo de fluidoterapia com solução fisiológica 0,9\%: G1 (sem fluidoterapia), G2 (uma hora de fluidoterapia antes da cisplatina), G3 (uma hora de fluidoterapia antes da cisplatina e uma hora após) e G4 (duas horas de fluidoterapia antes da cisplatina e uma após). Todos os animais receberam a cisplatina $\left(70 \mathrm{mg} / \mathrm{m}^{2}\right)$, pela via intravenosa, sendo os ciclos de quimioterapia realizados em intervalos de três semanas, num total de três ciclos. O ondansetron $(0,4 \mathrm{mg} / \mathrm{kg})$ foi administrado pela via intravenosa, a cada oito horas, no dia da quimioterapia e, a seguir, a cada 12 horas, por dois dias. O methimazole $(40 \mathrm{mg} / \mathrm{kg})$ foi pela via oral, 30 minutos antes da cisplatina e quatro horas após. Avaliaram-se os parâmetros hematológicos, bioquímicos, urinários e dosagem de tiroxina e triiodotironina a cada sete dias até o término do experimento. Esse protocolo foi eficaz e seguro em cães que permaneceram sob fluidoterapia durante duas a três horas. Os animais que não receberam fluidoterapia e os que ficaram somente uma hora sob infusão intravenosa de solução fisiológica apresentaram alterações que resultaram em nãorecomendação do protocolo.
\end{abstract}

Palavras-chave: cão, cisplatina, quimioterapia

\begin{abstract}
Twelve male dogs were divided in four experimental groups $(G)$, which were selected according to the time of $0.9 \%$ saline fluid therapy: G1 (without saline therapy), G2 (an hour of saline therapy before receiving cisplatin), G3 (an hour of saline therapy before receiving cisplatin and one hour after) and G4 (two hours of saline therapy before receiving cisplatin and one hour after). All the animals received $70 \mathrm{mg} / \mathrm{m}^{2}$ cisplatin, via intravenous, and the chemotherapy cycles were accomplished in intervals of three weeks, in a total of three cycles. Ondansetron $(0.4 \mathrm{mg} / \mathrm{kg})$ was administered via intravenous, every 8 hours, on the day of the chemotherapy and, from that, every 12 hours for two days. Methimazole $(40 \mathrm{mg} / \mathrm{kg})$ was orally administered 30 minutes before the cisplatin injection and four hours after. The hematological, biochemical and urinary parameters and dosages of tiroxin and triiodotironine were evaluated every seven days until the end of the experiment. This protocol was effective and safe in the animals that stayed for a period of two or three hours receiving saline therapy, because they did not present clinical and laboratory alterations of the use of chemotherapy. The animals that did not receive saline therapy and those that received infusion of physiologic solution via IV only during one hour presented alterations that made unfeasible the use of this protocol.
\end{abstract}

Keywords: dog, cisplatin, chemotherapy

Recebido em 28 de julho de 2005

Aceito em 7 de fevereiro de 2007

E-mail: claudiarepetti@yahoo.com.br. 


\section{INTRODUÇÃO}

$\mathrm{O}$ tratamento do câncer tem-se tornado rotineiro na clínica de pequenos animais. Isso se deve, provavelmente, a vários fatores, como o aumento da procura por parte dos proprietários de animais portadores de neoplasias e evidências de que a terapia, principalmente a quimioterapia, pode ser efetiva no controle tumoral, resultando em melhora na qualidade de vida e maior tempo de sobrevida (O'Keefe e Harris, 1990).

A cisplatina é um antineoplásico efetivo no tratamento adjuvante de osteossarcoma, carcinoma de células escamosas; tumores da bexiga, testículos, esôfago, e pulmão; melanomas e mesoteliomas (Bonassa, 2000; Sobral et al., 2001), sendo contra-indicada na espécie felina, pois pode causar edema pulmonar fulminante (Delisle et al., 1996). É o antineoplásico mais nefrotóxico usado em cães (Kisseberth e MacEwen, 1996).

Page et al. (1985) postularam algumas teorias sobre o mecanismo de nefrotoxicidade da cisplatina. A secreção tubular do fármaco envolve acúmulo da platina nas células epiteliais tubulares com transporte passivo para o lúmen. Esse acúmulo intracelular induz a disfunção tubular, promovendo depleção de energia e necrose. Outro mecanismo proposto é a ativação do sistema renina-angiotensina, o que resulta em diminuição na taxa de filtração glomerular e fluxo sangüíneo renal, induzindo o aparecimento de necrose tubular.

Apesar de existirem vários protocolos de diurese salina, o esquema de quatro horas de hidratação ainda é relativamente mais seguro para cães, consistindo na administração de solução fisiológica $0,9 \%$, por via intravenosa (IV), a $25 / \mathrm{kg} /$ hora, por três horas. Posteriormente, a cisplatina é administrada na dosagem máxima de $70 \mathrm{mg} / \mathrm{m}^{2} \mathrm{IV}$ por aproximadamente 20 minutos, usando-se esquema de infusão intravenosa lenta, seguido por administração de salina a $25 / \mathrm{kg} /$ hora, por mais uma hora. Esse protocolo deve ser repetido a cada três semanas (Ogilvie et al., 1993; Ogilvie, 1996). Náuseas e vômitos são graves e podem impor a suspensão do tratamento (Coppoc, 1992).

Methimazole, fármaco comumente utilizado no tratamento de hipertireoidismo, vem sendo usado na redução da toxicidade renal induzida pelos agentes antineoplásicos (Vail et al., 1993). Além de agir como antioxidante, esse fármaco restaura a defesa celular e bloqueia a peroxidação lipídica e, assim, protege contra a toxicidade de vários antineoplásicos nefrotóxicos (Elfarra et al., 1994).

Osman et al. (2000) revelaram que a cisplatina promove aumento significativo nos níveis renais de peróxidos lipídicos totais. $\mathrm{O}$ papel da peroxidação lipídica e sua posição na seqüência de eventos que induzem a nefrotoxicidade permanecem controversos. Hannemann e Baumann (1988) sugeriram que a geração de radicais livres e a subseqüente peroxidação lipídica são a causa da nefrotoxicidade.

O efeito protetor do methimazole na nefrotoxicidade induzida pela cisplatina foi estudado em cães por Vail et al. (1993). Os animais receberam $40 \mathrm{mg} / \mathrm{kg}$ methimazole, por via intraperitoneal, 30 minutos antes e quatro horas após a cisplatina, sem pré-hidratação com solução salina. Nesse estudo, o methimazole impediu o aumento do nitrogênio protéico e da concentração sérica de creatinina, além de reduzir o desenvolvimento de lesões histológicas renais após doses conhecidas desse quimioterápico como causadoras de tais lesões.

A toxicidade gastrointestinal é freqüente, e, muitas vezes, náuseas, anorexia e emese tornamse fatores limitantes da quimioterapia (Delisle et al., 1996). O papel da serotonina (5-HT) é de considerável interesse no processo emético, sendo identificados três receptores de 5-HT, sendo que o tipo três (5-HT3) apresenta papel primordial no processo de emese (Lichter, 1996).

Ondansetron é um antagonista seletivo de receptores 5-HT3, inicialmente desenvolvido para o tratamento de enxaqueca, sendo subseqüentemente aprovado, pela via oral ou intravenosa, na prevenção da emese induzida por agentes antineoplásicos (Köseoglu et al., 1998).

A quimioterapia com cisplatina induz a um padrão bifásico de emese, que é caracterizado por uma fase aguda e outra tardia. Seguindo o pico inicial de emese intensa, que ocorre dentro das primeiras oito horas após a quimioterapia, há um pico secundário de emese tardia, com náuseas e vômitos que ocorrem após 24 horas da 
administração da cisplatina (Kris et al., 1985). Com o controle da emese aguda induzida pelos agentes quimioterápicos, como a cisplatina, a emese tardia tem sido considerada como o maior problema clínico para pacientes recebendo quimioterapia (Kris et al., 1985). Apesar de a intensidade da emese tardia não ser tão grave quanto a emese aguda após a administração da cisplatina, ela é observada em todos os pacientes que não recebem terapia antiemética específica (Rudd e Naylor, 1997).

Berry et al. (1992), ao administrarem ondansetron a pacientes na dose de $0,15 \mathrm{mg} / \mathrm{kg}$ a cada quatro horas por três dias, iniciado 30 minutos antes da quimioterapia com cisplatina, observaram que a terapia com esse medicamento resultou em controle de náusea e vômito, com poucos efeitos colaterais, e proporcionou melhor qualidade de vida a esses pacientes em comparação com a terapia antiemética padrão.

Este trabalho teve o objetivo de avaliar um novo protocolo de quimioterapia e fluidoterapia com a cisplatina em cães.

\section{MATERIAL E MÉTODOS}

Utilizaram-se 12 cães, machos, com peso médio de 15 quilos, sem raça definida e divididos, aleatoriamente, em quatro grupos experimentais (G) de três animais cada um. Cada grupo foi selecionado conforme o tempo de fluidoterapia: G1 - sem fluidoterapia, G2 - uma hora de fluidoterapia antes da cisplatina, G3 - uma hora de fluidoterapia antes da cisplatina e uma hora depois e G4 - duas horas de fluidoterapia antes da cisplatina e uma depois.

Todos os animais receberam cisplatina ${ }^{1}$ na dose de $70 \mathrm{mg} / \mathrm{m}^{2}$, por via intravenosa (IV), diluída em solução fisiológica e administrada por um período de 20 minutos. Nesse protocolo, a fluidoterapia foi realizada com solução salina $0,9 \%$, numa velocidade de $25 \mathrm{ml} / \mathrm{kg} /$ hora. Os ciclos de quimioterapia foram realizados em intervalos de três semanas, totalizando três ciclos em todos os grupos.

\footnotetext{
${ }^{1}$ Cisplatina - Eli Lilly do Brasil Ltda. - São Paulo, Brasil
}

Todos os animais receberam ondansetron ${ }^{2}$ na dose de $0,4 \mathrm{mg} / \mathrm{kg}$ (IV), a cada oito horas, no dia da quimioterapia, começando 30 minutos antes da quimioterapia, e a cada 12 horas por dois dias. $\mathrm{O}$ methimazole $^{3}$ na dose de $40 \mathrm{mg} / \mathrm{kg}$ foi administrado por via oral, 30 minutos antes da cisplatina e quatro horas depois.

Por meio de venipunção cefálica, foram colhidos $2,0 \mathrm{ml}$ de sangue para realização de hemograma e plaquetometria. As amostras de urina foram obtidas por cateterismo vesical e analisadas no máximo 30 minutos após a colheita.

Foram colhidos 10,0ml de sangue, por venipunção da jugular externa, para realização dos seguintes exames séricos: creatinina, uréia, proteínas totais, albumina, alanina amino transferase, bilirrubinas direta e total e fosfatase alcalina.

Para determinação das concentrações de fósforo, potássio, cálcio, magnésio e glicose, as amostras foram processadas utilizando-se kits Labtest. Os valores de tiroxina (T4) e 3,5,3'-triiodotironina (T3) foram mensurados por meio de kits comerciais ${ }^{4}$. Essas análises foram realizadas imediatamente antes do primeiro ciclo de quimioterapia e, depois, a cada sete dias, num total de 10 análises.

Todos os animais foram avaliados clinicamente durante todo o período experimental.

Para as variáveis quantitativas, utilizou-se da análise de variância de medidas repetidas, com dois fatores, grupo (com quatro níveis) e ciclo (com três níveis), entre os animais e um fator tempo (com quatro níveis), dentro de animais. Foram utilizados três animais por grupo. Aplicou-se o teste t de Student nas comparações múltiplas (Zar, 1999). Para as análises qualitativas, usou-se o teste exato de Fisher (independência) (Zar, 1999).

Os momentos de amostragem para análise dos dados do eritrograma, leucograma, plaquetas, parâmetros bioquímicos e determinação hormonal de T3 e T4 foram classificados como:

\footnotetext{
${ }^{2}$ Zofran - Glaxo Welcome Ltda. - São Paulo, Brasil

${ }^{3}$ Tapazol - Eli Lilly do Brasil Ltda. - São Paulo - Brasil

${ }^{4}$ (Coat-a-count T3 e T4) - Diagnostic Products Coorporation -

Los Angeles, EUA
} 
M0 - amostragem inicial prévia ao primeiro ciclo de quimioterapia (controle); M1 - amostragem sete dias após o primeiro ciclo de quimioterapia; M2 - amostragem 14 dias após o primeiro ciclo de quimioterapia; e M3 - amostragem 21 dias após o primeiro ciclo de quimioterapia.

Após o segundo ciclo de quimioterapia: M4 - amostragem sete dias após o segundo ciclo de quimioterapia; M5 - amostragem 14 dias após o segundo ciclo de quimioterapia e M6 - amostragem 21 dias após o segundo ciclo de quimioterapia.

Depois do terceiro ciclo de quimioterapia: M7 - amostragem sete dias após o terceiro ciclo de quimioterapia; M8 - amostragem 14 dias após o terceiro ciclo de quimioterapia; e M9 - amostragem 21 dias após o terceiro ciclo de quimioterapia.

\section{RESULTADOS E DISCUSSÃO}

Os valores médios referentes ao número de hemácias, taxa de hemoglobina e hematócrito apresentaram-se dentro da normalidade durante todo o período experimental, não havendo diferença significativa entre os grupos. Com relação à contagem de plaquetas, durante todo o período experimental não houve diferença entre os grupos, com os valores dentro dos parâmetros da normalidade (Fig. 1).

A contagem das formas leucocitárias absolutas revelou que os diferentes grupos apresentaram diferenças entre os protocolos de tratamento, para os valores das contagens globais de leucócitos, bastonetes, neutrófilos segmentados e eosinófilos. No entanto, os valores globais apresentaram-se dentro dos limites da normalidade.

Com relação ao quadro hematológico dos pacientes tratados, não se observaram alterações que pudessem ser atribuídas ao uso dos protocolos utilizados. Esses dados estão de acordo com os citados por Bonassa (2000), em que a mielossupressão causada pelo uso da cisplatina é moderada e clinicamente imperceptível e em que as linhagens de células progenitoras estão protegidas dos efeitos da cisplatina, porque estão na fase G0 do ciclo celular. Desse modo, essas células podem ser recrutadas a entrar em divisão celular, permitindo que a medula se recupere após a remoção do agente quimioterápico.

Ogilvie et al. (1993) observaram diminuição significativa na contagem global de neutrófilos ao utilizarem protocolo de diurese com administração de uma hora de solução salina. Segundo eles, doses repetidas de cisplatina podem ter impedido a recuperação completa da medula, antes de cada ciclo de quimioterapia.

O'Keefe e Harris (1990) citaram que o grau de mielossupressão pode ser influenciado pelo mecanismo de ação e pela especificidade ciclocelular do quimioterápico. Segundo os autores, quimioterápicos ciclocelulares não específicos, como a cisplatina, tendem a causar citopenias rápidas, discretas e com rápida recuperação.

Quanto ao pH urinário, não houve diferença entre grupos em nenhum momento do período experimental. Pela análise qualitativa, e utilizando o teste de Fisher, verificou-se diferença significativa entre os grupos com relação à presença da proteína na urina, pois os animais de G1 e G2 apresentaram proteinúria em todos os momentos do período experimental (Fig. 2). Os demais parâmetros físico-químicos e de sedimentoscopia da urina se revelaram dentro dos valores da normalidade em todos os momentos do período experimental.

Os menores valores de densidade urinária observados nos animais de G1 e G2 (Fig. 3) durante o período experimental caracterizam perda da capacidade funcional dos rins em concentrar a urina.

Os resultados da urinálise obtidos em G3 e G4 evidenciaram que o protocolo de quimioterapia com a cisplatina mostrou-se efetivo para prevenir lesões renais sem comprometimento da função dos rins. A capacidade para concentrar urina foi mantida, como revelado pela densidade urinária. 


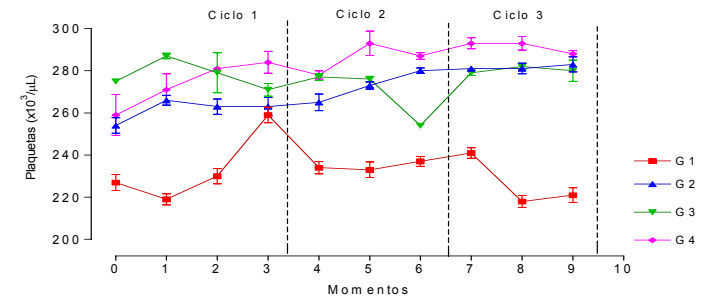

Figura 1. Valores médios da variável plaquetas $\left(\mathrm{x} 10^{3} / \mu \mathrm{l}\right)$ em cães submetidos a diferentes protocolos de fluidoterapia utilizando a cisplatina. $\mathrm{M} 0=$ início; M1, M2, M3 = 7, 14 e 21 dias após o primeiro ciclo de quimioterapia, respectivamente; M4, M5 e M6 $=7,14$ e 21 dias após o segundo ciclo de quimioterapia, respectivamente; e M7, M8 e M9 = 7, 14 e 21 dias após o terceiro ciclo de quimioterapia, respectivamente.

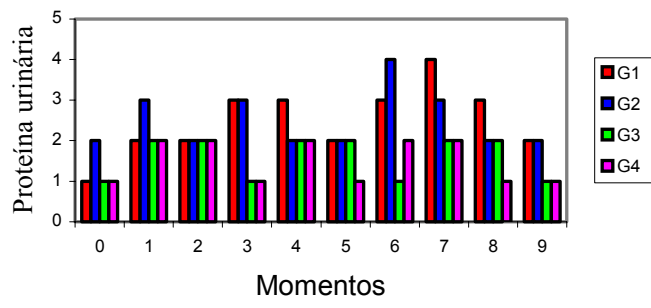

Figura 2. Valores médios da variável proteína urinária em cães submetidos a diferentes protocolos de fluidoterapia utilizando a cisplatina. $1=$ ausência de proteína na urina; $2=$ positivo $(+) ; 3=$ positivo $(++)$; e $4=$ positivo $(+++)$

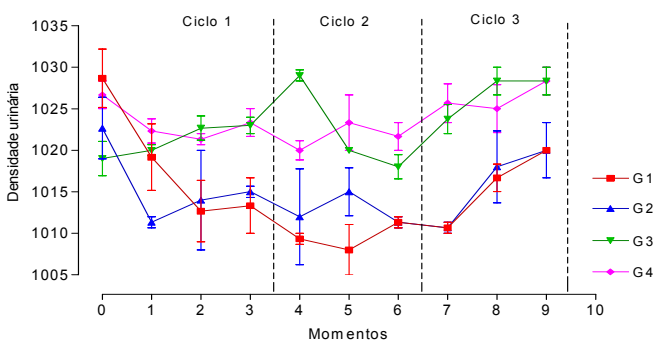

Figura 3. Valores médios e erro-padrão da variável densidade urinária em cães submetidos a diferentes protocolos de fluidoterapia utilizando a cisplatina.

Observaram-se elevações nos valores médios obtidos para uréia em G1 e G2 em relação aos demais, havendo diferenças significativas em todos os momentos do período experimental (Fig. 4). Houve diferença entre os valores médios de creatinina, sendo os animais de G1 e G2 os que apresentaram os valores mais elevados (Fig. 5).

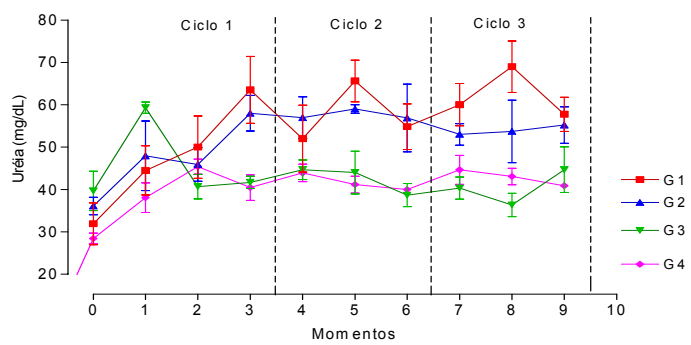

Figura 4. Valores médios e erro padrão da variável uréia $(\mathrm{mg} / \mathrm{dl})$ em cães submetidos a diferentes protocolos de fluidoterapia utilizando a cisplatina.

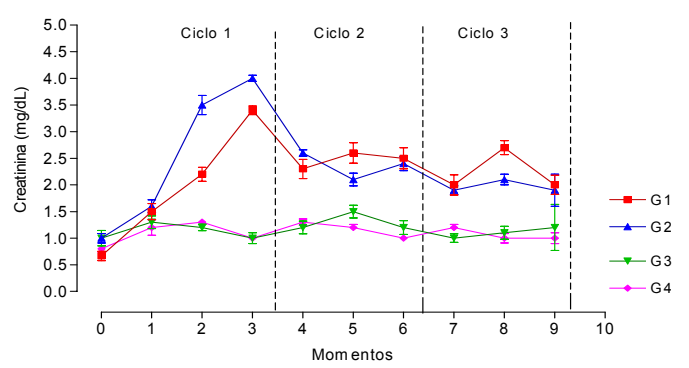

Figura 5. Valores médios e erro padrão da variável creatinina $(\mathrm{mg} / \mathrm{dl})$ em cães submetidos a diferentes protocolos de fluidoterapia utilizando a cisplatina.

Os maiores valores de creatinina sérica dos animais de G1 e G2 indicaram que a cisplatina induziu nefrotoxicidade, por diminuir a taxa de filtração glomerular e o fluxo sangüíneo renal e também por lesão direta nos túbulos renais, conforme discutido por Ogilvie et al. (1992). Segundo os autores, a queda na taxa de filtração glomerular secundária à administração da cisplatina pode resultar de vários mecanismos, incluindo ativação do sistema reninaangiotensina-aldosterona, que induz necrose tubular.

Os animais que apresentaram elevações nas concentrações séricas de uréia e creatinina foram os do G1 e G2. Isso permite discutir a presença de um metabólito da cisplatina como responsável por essa lesão renal. A cisplatina em um ambiente com alta concentração de cloreto não é tóxica e torna-se capaz de atravessar a membrana celular. Quando o quimioterápico entra na célula, ele se torna carregado positivamente, pois os grupos cloretos do quimioterápico são substituídos por radicais hidroxila ou água. Nesse estado positivamente carregado, a 
cisplatina torna-se citotóxica, reagindo com o DNA celular. Quando o processo de hidroxilação ocorre no meio extracelular, o potencial de nefrotoxicose aumenta, justificando a necessidade de se assegurar hidratação adequada com solução salina para manter a cisplatina numa estrutura neutra (Ogilvie et al., 1993).

A inclusão do methimazole ao protocolo de quimioterapia com a cisplatina justifica-se pelo fato de haver um grupo de pesquisadores que acredita que a lesão renal causada por esse quimioterápico se deva à formação de radicais livres nos rins (Vail et al., 1994; Osman et al., 2000). Vários estudos (Elfarra et al., 1994; Osman et al., 2000) têm evidenciado que a associação do methimazole à quimioterapia com cisplatina promove redução das concentrações séricas dos produtos nitrogenados não protéicos e previne a ocorrência de lesões renais após doses de cisplatina conhecidas por causar lesão tubular.

Neste estudo não se obteve proteção completa contra a nefrotoxicidade induzida pela cisplatina, ao se utilizar o methimazole, diferente do que foi citado por Osman et al. (2000). Talvez tenha sido a via de administração utilizada a responsável pela ineficiência do protocolo em proteger contra lesões renais, em todos os grupos experimentais. Observou-se que os animais de G1 e G2 apresentaram lesões renais graves, mesmo recebendo a mesma dosagem de methimazole que os de G3 e G4, que não apresentaram lesão renal. Os animais de G1 e G2 foram os que receberam menores volumes de fluidos, o que também pode ter influenciado o aparecimento de tais lesões renais, conforme citado por Ogilvie et al. (1993).

Com relação às proteínas totais e à albumina, não foram observadas diferenças significativas entre os grupos tratados, pois a maioria dos valores permaneceu dentro dos padrões da normalidade.

Não se observaram alterações nos valores séricos da fosfatase alcalina, da alanina aminotransferase e da bilirrubina total ou direta que pudessem estar relacionadas ao efeito citostático da cisplatina no sistema hepático dos animais submetidos a diferentes protocolos de fluidoterapia com o quimioterápico. De fato, nenhum autor mencionou o efeito hepatotóxico induzido pela terapia antineoplásica com a cisplatina. Do mesmo modo, os eletrólitos cálcio, fósforo, magnésio e potássio permaneceram dentro dos valores da normalidade durante todo o período experimental, assim como os valores da glicose plasmática, demonstrando que a quimioterapia com cisplatina não altera a glicemia dos animais.

Os resultados deste trabalho confirmam os citados por Vail et al. (1994), segundo os quais a administração do methimazole não provoca declínio nas concentrações de T3 e T4. Como a glândula tireóide contém hormônio estocado no interior dos ácinos, curtos períodos de administração de methimazole não alteram os padrões dos hormônios tireoidianos.

Observou-se, em todos os animais de G1, a ocorrência de episódios eméticos, após a segunda administração das cápsulas de methimazole, o que ocorreu quatro horas após o término da quimioterapia. Somente um animal de G2 apresentou emese após receber o methimazole, e em nenhum dos demais grupos isso ocorreu. Como os animais já podiam apresentar náuseas em decorrência da quimioterapia adotada, pode ser que a presença do medicamento no trato gastrintestinal tenha sido suficiente para induzir o vômito, conseqüentemente, não se obteve o efeito esperado.

Nas condições em que se desenvolveu este experimento, o uso do ondansetron foi efetivo no controle de episódios eméticos, tanto agudos quanto tardios, dos animais de G3 e G4. Esses resultados estão em acordo com os citados por Köseoglu et al. (1998), que consideraram o ondansetron $o$ antiemético de escolha para pacientes portadores de neoplasias, que receberam quimioterapia com cisplatina.

Não se observou efetividade do ondansetron no controle dos episódios de emese aguda nos animais de G1, diferente do observado por Rudd e Naylor (1997), que relataram que a eficácia dos antagonistas de receptores 5-HT3 é menor no caso de vômitos que ocorrem em longo prazo, em comparação com episódios eméticos agudos. Porém, tem-se que levar em consideração o fato de esses animais terem recebido methimazole pela via oral, o que pode ter contribuído para a ocorrência de tais episódios eméticos. Além disso, esses animais não receberam fluidoterapia (G1) ou receberam somente uma hora de fluidoterapia (G2), podendo levar em 
consideração a importância de se realizar a hidratação adequada do paciente (Ogilvie et al., 1993).

De acordo com o esquema de administração do ondansetron deste experimento, conseguiram-se controlar, em todos os grupos, os episódios eméticos em longo prazo, que se constituem num dos maiores desafios para os oncologistas clínicos, como citado por Kris et al. (1985).

uQuanto ao apetite, observou-se que os animais de G1 apresentaram diminuição na ingestão de alimentos no dia seguinte aos ciclos de quimioterapia. Ressalta-se que os demais mantiveram a ingestão de ração normal ao longo de todo o período experimental.

\section{CONCLUSÕES}

A cisplatina pode ser usada com segurança em animais sem doença renal prévia, associando-se ondansetron e methimazole a um protocolo de uma hora de fluidoterapia antes da cisplatina e uma hora após ou a um protocolo de duas horas de fluidoterapia antes da cisplatina e uma hora depois. O methimazole, administrado pela via oral, é seguro na prevenção da nefrotoxicidade induzida pela cisplatina utilizando um protocolo de duas ou três horas de fluidoterapia, assim como o ondansetron é um antiemético seguro na prevenção da emese, em longo prazo, induzida pela cisplatina.

\section{REFERÊNCIAS BIBLIOGRÁFICAS}

BERRY, W.R.; HOUSE, K.W.; LEE, J.T. et al. Results of a compassionate-use program using intravenous ondansetron to prevent nausea and vomiting in patients receiving emetogenic cancer chemotherapy. Sem. Oncol., v.19, suppl.15, p.3337, 1992.

BONASSA, E.M.A. Conceitos gerais em quimioterapia antineoplásica. In: Enfermagem em quimioterapia. São Paulo: Atheneu, 2000. p.3-20.

COPPOC, G.L. Quimioterapia das doenças neoplásicas. In: BOOTH, N.H.; McDONALD, L.E. Farmacologia e terapêutica veterinária. 6.ed. Rio de Janeiro: Guanabara Koogan, 1992. p.693-706.
DELISLE, F.; DEVAUCHELLE, P.; DOLIGER, S. Cancérologie - Le cisplatine. Point Vet., v.28, p.69-71, 1996.

ELFARRA, A.A.; DUESCHER, R.J.; SAUSEN, P. J. et al. Methimazole protection of rats against gentamicin-induced nephrotoxicity. Can. J. Physiol. Pharmacol., v.72, p.1238-1244, 1994.

HANNEMANN, J.; BAUMANN, K. Cisplatininduced lipid peroxidation and decrease of gluconeogenesis in rat kidney cortex: different effect of antioxidant and radical scavengers. Toxicology, v.51, p.119-132, 1988.

KISSEBERTH, W.C.; MacEWEN, E.G. Complications of cancer and its treatment. In: WITHROW, S.J.; MacEWEN, E.G. Small animal clinical oncology. 2.ed. Philadelphia: W.B. Saunders, 1996. p. 70-86.

KÖSEOGLU, V.; KÜREKÇI, A.E.; SARICI, U. et al. Comparison of the efficacy and side effects of ondansetron and metoclopramidediphenhydramine administered to control nausea and vomiting in children treated with antineoplastic chemotherapy: a prospective randomized study. Eur. J. Pediatr., v.157, p.806810,1998 .

KRIS, M.G.; GRALLA, R.J.; CLARK, R.A. et al. Incidence, course, and severity of delayed nausea and vomiting following the administration of high-dose cisplatin. J. Clin. Oncol., v.3, p. 1379-1384, 1985.

LICHTER, I. Nausea and vomiting in patients with cancer. Hematol. Oncol. Clin. N. Am., v.10, p.207-220, 1996.

OGILVIE, G.K.; FETTMAN, M.J.; JAMESON, V.J. et al. Evaluation of one hour saline diuresis protocol for administration of cisplatin to dogs. Am. J. Vet. Res., v.23, p.1666-1669, 1992.

OGILVIE, G.K.; STRAW, R.C.; JAMESON, V.J. et al. Prevalence of nephrotoxicosis associated with a four-hour saline diuresis protocol for the administration of cisplatin to dogs with naturally developing neoplasms. $J$. Am. Vet. Med. Assoc., v.202, p.1845-1848, 1993.

OGILVIE, G.K. Chemotherapy. In: WITHROW, S.J.; MacEWEN, E.G. Small animal clinical oncology. 2.ed. Philadelphia: W.B. Saunders, 1996. p.70-86. 
O'KEEFE, D.A.; HARRIS, C.L. Toxicology of oncologic drugs. Vet. Clin. N. Am.: Small Anim. Pract., v.20, p.483-504, 1990.

OSMAN, A.M.; EL-SAYED, E.M.; ELDEMERDASH, E. et al. Prevention of cisplatininduced nephrotoxicity by methimazole. Pharmacol. Res., v.41, p.115-121, 2000.

PAGE, R.L.; MATUS, R.E.; LEIFER, C.E. et al. Cisplatin, a new antineoplastic drug in veterinary medicine. J. Am. Vet. Med. Assoc., v.186, p.288290, 1985.

RUDD, J.A.; NAYLOR, R.J. the actions of ondansetron and dexamethasone to antagonize cisplatin-induced emesis in the ferret. Eur. J. Pharmacol., v.322, p.79-82, 1997.
SOBRAL, R.A.; DALECK, C.R.; MELO, W.J. Estudo in vivo de um sistema de liberação de drogas constituído por hidroxiapatita e cisplatina. Acta Cir. Bras., v.16, p.128-132, 2001.

VAIL, D.N.; ELFARRA, A.A.; COOLEY, A.J. et al. Methimazole as a protectant against cisplatin-induced nephrotoxicity using the dog as a model. Cancer Chemother. Pharmacol., v.33, p.25-30, 1993.

VAIL, D.M.; ELFARRA, A.A.; PANCIERA, D.0L. et al. Pharmakinetics and short-term clinicopathologic changes after intravenous administration of a high dose of methimazole in dogs. Am. J. Vet. Res., v.55, p.1597-1601, 1994.

ZAR, J.H. Biostatistical analysis. 4.ed. New Jersey: Prentice Hall, 1999. 663p. 